\title{
New tools and simpler algorithms for branchwidth
}

\author{
Christophe Paul ${ }^{1}$ and Jan Arne Telle ${ }^{2}$ \\ 1 CNRS - LIRMM, Montpellier, France, paul@lirmm.fr \\ 2 Department of Informatics, University of Bergen, Norway \\ (visiting LIRMM 2004-05), telle@ii.uib.no
}

\begin{abstract}
We provide new tools, such as $k$-troikas and good subtreerepresentations, that allow us to give fast and simple algorithms computing branchwidth. We show that a graph $G$ has branchwidth at most $k$ if and only if it is a subgraph of a chordal graph in which every maximal clique has a $k$-troika respecting its minimal separators. Moreover, if $G$ itself is chordal with clique tree $T$ then such a chordal supergraph exists having clique tree a minor of $T$. We use these tools to give a straightforward $O\left(m+n+q^{2}\right)$ algorithm computing branchwidth for an interval graph on $m$ edges, $n$ vertices and $q$ maximal cliques. We also prove a conjecture of F. Mazoit [12] by showing that branchwidth is polynomial on a chordal graph given with a clique tree having a polynomial number of subtrees.
\end{abstract}

\section{Introduction}

Branchwidth and treewidth are connectivity parameters of graphs and whenever one of these parameters is bounded by some fixed constant on a class of graphs, then so is the other [13]. Since many graph problems that are in general NPhard can be solved in linear time on such classes of graphs both treewidth and branchwidth have played a large role in many investigations in algorithmic graph theory. Recently there has been a focus on branchwidth $[6,5,4,7,8]$ to give e.g. good heuristics for the travelling salesman problem and fast parameterized algorithms for various types of optimization problems. These algorithms always involve a stage that constructs a branch-decomposition with small branchwidth, and another stage solving the problem using the decomposition by a running time depending heavily on that branchwidth. Efficient algorithms computing optimal branch-decompositions, like we give in this paper, could therefore be the crucial factor that can make or break the application.

The study of branchwidth has not enjoyed the rich toolbox that treewidth has with its connections to $k$-trees, chordal graphs of maximum clique size, intersection graphs of subtrees of a tree etc. We try to rectify this in the current paper, by introducing various new tools like $k$-troikas, $k$-good chordal graphs and good subtree representations, whose definitions will follow later. To give an example using only standard terminology, we remark that using these tools we arrive at a succinct expression of the common basis of treewidth and branchwidth: For any $k \geq 2$ a graph $G$ on vertices $v_{1}, v_{2}, \ldots, v_{n}$ has branchwidth at most $k$ (treewidth at most $k-1)$ if and only if there is a cubic tree $T$ with subtrees $T_{1}, T_{2}, \ldots, T_{n}$ such that if $v_{i}$ and $v_{j}$ adjacent then subtrees $T_{i}$ and $T_{j}$ share at least one edge (node) of $T$, and each edge (node) of $T$ is shared by at most $k$ of the subtrees (replace underlined words by the words in parenthesis.) 
The understanding of branchwidth of special graph classes is relatively limited. We give a brief overview of the literature. In a paper from 1994 Seymour and Thomas showed that branchwidth is NP-complete in general, and followed this by their celebrated ratcather method computing branchwidth of planar graphs in polynomial time [14]. In 1997 Bodlaender and Thilikos used fairly brute-force methods to give a linear-time algorithm deciding if a graph has branchwidth at most some constant $k$ [1] and a very elegant algorithm for graphs of branchwidth 3 [2]. Then in 1999 Kloks, Kratochvil and Müller [11] pushed into new territory by showing that branchwidth is NP-complete already for split graphs and bipartite graphs, with the bulk of their paper being an $O\left(n^{3} \log n\right)$ algorithm for branchwidth of interval graphs with the comment that "it is somewhat surprising that this algorithm is by no means straightforward and its correctness proof requires a nontrivial proof." In contrast, using our branchwidth tools for the case of interval graphs we arrive at a straightforward $O\left(n^{2}\right)$ algorithm whose self-contained correcteness proof is easy to follow. In fact, our algorithm has runtime $O\left(m+n+q^{2}\right)$ for an interval graph on $m$ edges, $n$ vertices and $q$ maximal cliques. In a recent investigation Mazoit gave a polynomial-time algorithm for branchwidth of circulararc graphs and conjectured that branchwidth can be computed in polynomial-time for chordal graphs given with a clique tree having a polynomial number of subtrees [12]. We prove his conjecture in this paper. Indeed, it follows by a generalization of the interval graph algorithm since we show a structural property stating that branchwidth of a chordal graph with clique tree $T$ can be found by considering chordal supergraphs whose clique tree is a minor of $T$.

In Section 2 we give some standard definitions. In Section 3 we use subtreerepresentations to characterize graphs of branchwidth $k$ as subgraphs of chordal graphs. In Section 4 we study the central new concept of $k$-troikas in a purely settheoretic setting. In Section 5 we give a simple algorithm computing branchwidth for interval graphs and more generally for chordal graphs with a clique tree having a polynomial number of subtrees.

\section{Standard definitions}

We consider simple undirected and connected graphs $G$ with vertex set $V(G)$ and edge set $E(G)$. We denote $G$ subgraph of $H$ by $G \subseteq H$ which means that $V(G)=V(H)$ and $E(G) \subseteq E(H)$. For a set $A \subseteq V(G), G(A)$ denotes the subgraph of $G$ induced by the vertices in $A$. $A$ is called a clique if $G(A)$ is complete. The set of neighbors of a vertex $v$ in $G$ is $N(v)=\{u \mid u v \in E(G)\}$. A vertex set $S \subset V(G)$ is a separator if $G(V(G) \backslash S)$ is disconnected. Given two vertices $u$ and $v, S$ is a $u, v$-separator if $u$ and $v$ belong to different connected components of $G(V(G) \backslash S)$. A $u, v$-separator $S$ is minimal if no proper subset of $S$ separates $u$ and $v$. In general, $S$ is a minimal separator of $G$ if there exist two vertices $u$ and $v$ in $G$ such that $S$ is a minimal $u, v$-separator. A graph is chordal if it contains no induced cycle of length $\geq 4$. In a clique tree of a chordal graph $G$ the nodes are in 1-1 correspondence with the maximal cliques of $G$ and the set of nodes whose maximal cliques contain a given vertex form a subtree. For further terminology, see e.g. [10]. We usually refer to nodes of a tree and vertices of a graph.

A branch-decomposition $(T, \mu)$ of a graph $G$ is a tree $T$ with nodes of degree one and three only, together with a bijection $\mu$ from the edge-set of $G$ to the set of 
degree-one nodes (leaves) of $T$. For an edge $e$ of $T$ let $T_{1}$ and $T_{2}$ be the two subtrees resulting from $T \backslash\{e\}$, let $G_{1}$ and $G_{2}$ be the graphs induced by the edges of $G$ mapped by $\mu$ to leaves of $T_{1}$ and $T_{2}$ respectively, and let $\operatorname{mid}(e)=V\left(G_{1}\right) \cap V\left(G_{2}\right)$. The width of $(T, \mu)$ is the size of the largest $\operatorname{mid}(e)$ thus defined. For a graph $G$ its branchwidth $b w(G)$ is the smallest width of any branch-decomposition of $G$.

NB: The graphs of branchwidth 1 are the stars, and constitute a somewhat pathological case. To simplify we therefore restrict attention to graphs having branchwidth $k \geq 2$, in other words our statements are correct only for graphs having at least two vertices of degree more than one.

\section{Good subtree-representations}

Definition 1. A subtree-representation $R=\left(T,\left\{T_{1}, T_{2}, \ldots, T_{n}\right\}\right)$ is a pair where $T$ is a tree with vertices of degree at most three and $T_{1}, T_{2}, \ldots, T_{n}$ are subtrees of $T$. Its edge intersection graph $E I(R)$ has vertex set $\left\{v_{1}, v_{2}, \ldots, v_{n}\right\}$ and edge set $\left\{v_{i} v_{j}: T_{i}\right.$ and $T_{j}$ share an edge of $\left.T\right\}$, while its vertex intersection graph $V I(R)$ has the same vertex set but edge set $\left\{v_{i} v_{j}: T_{i}\right.$ and $T_{j}$ share a node of $\left.T\right\}$. For a node $u$ of $T$, we call the set of vertices $X_{u}=\left\{v_{i}: T_{i}\right.$ contains $\left.u\right\}$ the bag of $u$, and $\left\{X_{u}: u \in V(T)\right\}$ the bags of $R$.

With the above terminology we can easily move between the view of a subtreerepresentation $R$ as a tree $T$ with a set of subtrees $\left\{T_{1}, T_{2}, \ldots, T_{n}\right\}$ or as a tree $T$ with a set of bags $\left\{X_{u}: u \in V(T)\right\}$. When manipulating the latter we must simply ensure that for any vertex in $E I(R)$ the set of bags containing that vertex corresponds to a set of nodes of $T$ inducing a subtree, i.e. a connected subgraph.

Definition 2. The edge-weight of subtree-representation $R=\left(T,\left\{T_{1}, T_{2}, \ldots, T_{n}\right\}\right)$ is the maximum, over all edges uv of $T$, of the number of subtrees in $\left\{T_{1}, T_{2}, \ldots, T_{n}\right\}$ that contain edge uv. $R$ is a good subtree-representation if $E I(R)=V I(R)$.

We are in this paper only interested in the edge intersection graphs of subtreerepresentations having bounded edge-weight $k$. We start by showing that we can restrict ourselves to good subtree-representations if we want.

Lemma 1. For any subtree-representation $R$ of edge-weight $k$ there exists a good subtree-representation $R^{\prime}$ of edge-weight $k$ with $E I(R)=E I\left(R^{\prime}\right)=V I\left(R^{\prime}\right)$.

Proof: Assume some bag $X_{i}$ of $R$ contains two vertices $a, b$ with $a b$ not an edge of $E I(R)$, meaning that no neighbor $j$ of $i$ has $\{a, b\} \subseteq X_{j}$. Since $i$ has at most three neighbors, this means that one of $a$ or $b$, say $a$, is in the bag of only one of these neighbors, say $j$. We subdivide the edge $i j$ in the tree $T$ with the new node having bag $X_{i} \cap X_{j}$, and remove $a$ from $X_{i}$. Repeat this procedure until the resulting subtree-representation $R^{\prime}$ is good. When we are done $R^{\prime}$ has edge-weight $k$ with $E I(R)=E I\left(R^{\prime}\right)=V I\left(R^{\prime}\right)$.

Lemma 2. A graph $G$ has branchwidth at most $k \Leftrightarrow$ there is a good subtreerepresentation $R$ of edge-weight at most $k$ with $G \subseteq E I(R)$. 
Proof: $\Rightarrow$ : Take a branch-decomposition $(T, \mu)$ of $G$ of width $k$, i.e. with $|\operatorname{mid}(e)| \leq$ $k$ for each $e \in E(T)$. We construct a subtree-representation $R=\left(T^{\prime}, S\right)$ of edgeweight $k$ with $G \subseteq E I(R)$. $T^{\prime}$ is constructed from $T$ by for each leaf $l$ of $T$ adding a new leaf $l^{\prime}$ and making it adjacent to $l$. For vertex $a \in V(G)$ consider the smallest spanning subtree of $T$ containing all leaves of $T$ that are mapped by $\mu$ to an edge incident with $a$. The subtree $T_{a}$ will be this subtree augmented by leaf $l^{\prime}$ for each leaf $l$ of $T$ that it contains. This completes the description of $R=\left(T^{\prime},\left\{T_{a}: a \in V(G)\right\}\right.$. For any two adjacent vertices $\{a, b\}$ of $G$ we have $\mu^{-1}(l)=\{a, b\}$ for some leaf $l$ of $T$, and thus the subtrees corresponding to $a$ and $b$ share the edge $l l^{\prime}$ of $T^{\prime}$ which implies that $G \subseteq E I(R)$. If vertex $a$ has subtree $T_{a}$ containing edge $e$ of $T$, then there are edges incident with $a$ mapped to leaves in both subtrees of $T$ arising from deleting the edge $e$, and thus $a \in \operatorname{mid}(e)$. But this means that the edge-weight of $R$ is at most $k$. If $R$ is not good then we can make it good by applying Lemma 1 .

$\Leftarrow$ : Let $R=(T, S)$ be a good subtree-representation $R$ of edge-weight at most $k$ with $G \subseteq E I(R)$. We construct a branch-decomposition $\left(T^{\prime}, \mu\right)$ of $G$ with width $k$. Associate each edge $a b$ of $G$ with an edge $e$ of $T$ such that the subtrees $T_{a}$ and $T_{b}$ corresponding to $a$ and $b$ both contain $e$. Subdivide the tree edge $e$ by as many new nodes as there are edges of $G$ associated to $e$, thus creating for each edge $a b$ associated to $e$ a new tree node $e_{a b}$. Furthermore, add a new leaf node $l_{a b}$, make it adjacent to $e_{a b}$ and set $\mu(a b)=l_{a b}$. Let $T^{\prime \prime \prime}$ be the tree we have constructed so far. It contains $T$ as a minor. Consider the smallest spanning subtree $T^{\prime \prime}$ of $T^{\prime \prime \prime}$ having the set of leaves $\left\{l_{a b}: a b \in E(G)\right\}$. Iteratively contract edges of $T^{\prime \prime}$ incident to a vertex of degree two until all inner vertices have degree three. The resulting tree is $T^{\prime}$. Note that as we constructed $T^{\prime}$ from $T$ in stages we could at each stage have updated the subtree $T_{a}$ corresponding to vertex $a$ to a new subtree $T_{a}^{\prime}$ so that we would still have a subtree-representation $R^{\prime}=\left(T^{\prime}, S^{\prime}\right)$ with $G \subseteq E I\left(R^{\prime}\right)$. For example, $T_{a}^{\prime}$ should contain every 'subdivision node' on a tree edge $f$ if $T_{a}$ contained $f$, it should contain $l_{a b}$ for any edge $a b$ incident with $a$, and it should naturally shrink if it contained a removed leaf or contracted edge. Moreover, $\left(T^{\prime}, S^{\prime}\right)$ has edge-weight at most $k$ since never during this process did we increase the edge-weight beyond what it was. $T^{\prime}$ has nodes of degree one and three only and $\mu$ is a bijection between its leaves and the edges of $G$ so $\left(T^{\prime}, \mu\right)$ is a branchdecomposition of $G$. It remains to show that it has width $k$, i.e. that for any edge $e$ of $T^{\prime}$ we have $|\operatorname{mid}(e)| \leq k$. We claim that $\operatorname{mid}(e) \subseteq\left\{a: T_{a}^{\prime}\right.$ contains edge $\left.e\right\}$. Consider $a \in \operatorname{mid}(e)$. There must exist two leaves $l_{a b}, l_{a c}$ of $T^{\prime}$, one in each of the two subtrees of $T^{\prime} \backslash e$, such that $a \in \mu^{-1}\left(l_{a b}\right)$ and $a \in \mu^{-1}\left(l_{a c}\right)$. Since the subtree $T_{a}^{\prime}$ of $a$ contains both $l_{a b}$ and $l_{a c}$ it must also contain $e$.

We introduce the concept of $k$-troikas ${ }^{3}$ which is a central tool in our investigation of branchwidth.

Definition 3. $A$ k-troika $(A, B, C)$ of a set $X$ are 3 subsets of $X$, called the three parts, such that $|A| \leq k,|B| \leq k,|C| \leq k$, and $A \cup B=A \cup C=C \cup B=X$. $(A, B, C)$ respects $S_{1}, S_{2}, \ldots, S_{q}$ if any $S_{i}, 1 \leq i \leq q$ is contained in at least one of $A, B$ or $C$.

\footnotetext{
${ }^{3}$ A troika is a horse-cart drawn by three horses, and when the need arises any two of them should also be able to pull the cart
} 
Definition 4. A $k$-good chordal graph is a chordal graph in which every maximal clique $X$ has a $k$-troika respecting the minimal separators contained in $X$.

Theorem 1. A graph $G$ has branchwidth at most $k \Leftrightarrow G$ is subgraph of a $k$-good chordal graph

Proof: $\Rightarrow$ : By Lemma 2 there exists a good subtree-representation $R$ of edgeweight $k$ with $G \subseteq E I(R)=V I(R)$. Since $V I(R)$ is a vertex intersection graph of subtrees of a tree it is a chordal graph [9], and $H=E I(R)=V I(R)$ will indeed be our chordal graph $H$ having $G$ as a subgraph. By the Helly property of (vertex) intersection of subtrees of a tree, every maximal clique of $H$ is a bag $X_{u}$ for some node $u$ of the tree. If $\left|X_{u}\right| \leq k$ then it clearly has a $k$-troika respecting any subset, so let us assume $\left|X_{u}\right|>k$. Since any pair $a, b$ of nodes from $X_{u}$ is adjacent in $H$, we must have $\{a, b\}$ contained also in one of the neighboring bags. Let the intersection of $X_{u}$ and the bags of its three neighbors be $A, B$ and $C$. This means that any two of $A, B, C$ must have union $X_{u}$ since if for example $a \in X_{u}$ but $a \notin A \cup B$ then we would be forced to have $C=X_{u}$, since $C$ would have to contain $a$ and all its neighbors in $X_{u}$ contradicting the fact that $R$ has edge-weight $k$. Any minimal separator $S$ of the chordal graph $H$ is the intersection of two maximal cliques corresponding to two bags $X_{u}, X_{v}$. If we assume $A=X_{u} \cap X_{w}$, for $w$ the neighbor of $u$ on the path from $u$ to $v$ in $T$, then we have $S=X_{u} \cap X_{v} \subseteq A$ since the subtree corresponding to a vertex $a \in\left(X_{u} \cap X_{v}\right) \backslash A$ would be disconnected.

$\Leftarrow$ : Consider any clique tree of the $k$-good chordal graph $H$ containing $G$. In fact this can be viewed as a pair $R=(T, S)$ just as our subtree-representations with $H=V I(R)$ and every bag inducing a maximal clique of $H$, except that nodes of $T$ can have degree larger than 3 . We construct from this a subtreerepresentation $R^{\prime}=\left(T^{\prime}, S^{\prime}\right)$ of edge-weight $k$ with $G \subseteq H \subseteq E I\left(R^{\prime}\right)$ which by Lemma 2 and Lemma 1 will imply that $G$ has branchwidth at most $k$. Let $X$ be a maximal clique whose node in $T$ has $q$ neighbors corresponding to maximal cliques $Z_{1}, Z_{2}, \ldots, Z_{q}$, and let $(A, B, C)$ be the $k$-troika of $X$ respecting minimal separators $X \cap Z_{1}, \ldots, X \cap Z_{q}$. This means there exists a partition $P_{A}, P_{B}, P_{C}$ of $\{1,2, \ldots, q\}$ such that $X \cap Z_{i} \subseteq A$ for $i \in P_{A}, X \cap Z_{i} \subseteq B$ for $i \in P_{B}, X \cap Z_{i} \subseteq C$ for $i \in P_{C}$. For maximal clique $X$ we construct a ternary subtree as follows: We have a central node with bag $X$ adjacent to three paths: one path with $\max \left\{1,\left|P_{A}\right|\right\}$ bags $A$, one path with $\max \left\{1,\left|P_{B}\right|\right\}$ bags $B$ and one with $\max \left\{1,\left|P_{C}\right|\right\}$ bags $C$. For each $i \in\{1,2, \ldots, q\}$ we have a leaf-node with bag $X \cap Z_{i}$ as neighbor of a node on these paths, e.g. if $i \in P_{A}$ the leaf-node should be the neighbor of a node with bag $A$, if $i \in P_{B}$ then $B$, and if $i \in P_{C}$ then $C$, such that $q$ of the nodes on the 3 paths get one leaf each. (see Figure 3 in Appendix). Construct such a ternary subtree for each maximal clique $X$, i.e. for each node of $T$. Then, for each pair of maximal cliques $X, Y$ that are bags of two neighboring nodes in $T$ we identify the following two leaves into a single node: $X \cap Y$ in the subtree constructed for $X$ and $Y \cap X$ in the subtree constructed for $Y$. The resulting tree $T^{\prime}$ has no node of degree more than three and together with bags as indicated it forms the subtree-representation $R^{\prime}=\left(T^{\prime}, S^{\prime}\right) . R^{\prime}$ has edge-weight at most $k$ since any part of a $k$-troika has size at most $k$. We show that $H \subseteq E I\left(R^{\prime}\right)$. For any edge $a b \in E(H)$ we have $\{a, b\} \subseteq X$ for some maximal clique $X$. The $k$-troika $(A, B, C)$ of $X$ has the property that any vertex $a \in X$ must be in two out of $A, B, C$, so that we must have $\{a, b\}$ contained in one of $A, B$ or $C$. Thus the edge $a b$ is in $E I\left(R^{\prime}\right)$ and $H \subseteq E I\left(R^{\prime}\right)$. 


\section{$4 \quad k$-Troikas}

This section will be devoted to a study of the conditions under which a set $X$ has a $k$-troika respecting a given set of subsets. As with branchwidth, we restrict attention to the case $k \geq 2$. These conditions on the given sets, which will turn out to be testable by simple algorithms, will in conjunction with Theorem 1 be useful for designing algorithms computing branchwidth of graphs.

Observation 1 If $X$ has a $k$-troika respecting $S_{1}, S_{2}, \ldots, S_{q}$ then $\left|S_{i}\right| \leq k$ for each $1 \leq i \leq q$ and $|X| \leq\lfloor 3 k / 2\rfloor$.

The above is obvious, every subset must be of size at most $k$ since it must be contained in a part of size at most $k$, and the fact that every pair of parts must have union $X$ means that every element of $X$ must belong to at least two parts which implies $2|X| \leq 3 k$. Note that the case of respecting a single subset is trivial, the necessary and sufficient conditions are that the subset has at most $k$ elements and $|X| \leq\lfloor 3 k / 2\rfloor$. Likewise, if $\left|S_{1} \cup S_{2} \cup \ldots \cup S_{q}\right| \leq k$ then $G$ has a $k$-troika respecting $S_{1}, S_{2}, \ldots, S_{q}$ precisely when $|X| \leq\lfloor 3 k / 2\rfloor$ since we may as well view the union of all the subsets as a single subset.

\section{1 $k$-Troikas respecting two subsets}

In this section we consider conditions under which a set $X$ has a $k$-troika respecting two subsets $S_{1}, S_{2}$. As mentioned above we assume that $\left|S_{1} \cup S_{2}\right|>k$ and also wlog that any $k$-troika $(A, B, C)$ respecting $S_{1}, S_{2}$ has $S_{1} \subseteq A$ and $S_{2} \subseteq B$. Note that if $X$ has a $k$-troika respecting $S_{1}, S_{2}$ then it has one where no element of $X$ belongs to all three parts. This motivates the following definition.

Definition 5. A k-tripartition of a set $X$ is a partition of $X$ into three (disjoint) partition classes, such that the sum of sizes of any two partition classes is at most k. A k-tripartition $\left(T_{1}, T_{2}, T_{3}\right)$ of $X$ respects $S_{1}, S_{2}$ if $S_{1} \cap S_{2} \subseteq T_{3}, S_{1} \subseteq T_{1} \cap T_{3}$, and $S_{2} \subseteq T_{2} \cap T_{3}$.

Observation 2 If $\left(T_{1}, T_{2}, T_{3}\right)$ is a k-tripartition of $X$ then $\left(T_{1} \cup T_{3}, T_{2} \cup T_{3}, T_{2} \cup T_{1}\right)$ is a $k$-troika of $X$, and the former respects $S_{1}, S_{2}$ iff the latter does. Conversely, if $(A, B, C)$ is a k-troika of $X$ with $A \cap B \cap C=\emptyset$ then $(A \cap C, B \cap C, B \cap A)$ is a k-tripartition of $X$, and the former respects $S_{1}, S_{2}$ iff the latter does (assuming $\left|S_{1} \cup S_{2}\right|>k$ as discussed above).

In view of this observation, when it comes to $k$-troikas respecting two subsets $S_{1}, S_{2}$ we need only consider those that arise from $k$-tripartitions. In Observation 1 we gave some obviously necessary conditions on $|X|,\left|S_{1}\right|,\left|S_{2}\right|$. What other necessary conditions do we have? Note that if $|X|=3 k / 2$ and $k$ is even then only a 'balanced' $k$-tripartition with each partition class having $k / 2$ vertices will do. Since we must have $S_{1} \cap S_{2} \subseteq T_{3}$ the case where $\left|S_{1} \cap S_{2}\right|>k / 2$ therefore implies a stronger size restriction on $X$. The best we could hope for is to set $T_{3}=S_{1} \cap S_{2}$ and put $k-\left|S_{1} \cap S_{2}\right|$ vertices into each of $T_{1}$ and $T_{2}$ which yields:

Observation 3 If $X$ has a $k$-troika respecting $S_{1}, S_{2}$ then $|X| \leq\left|S_{1} \cap S_{2}\right|+2(k-$ $\left.\left|S_{1} \cap S_{2}\right|\right)=2 k-\left|S_{1} \cap S_{2}\right|$ 
Note that we did not need to preface this observation by the condition "if $\left|S_{1} \cap S_{2}\right|>k / 2$ " since $|X| \leq\lfloor 3 k / 2\rfloor$ and $\left|S_{1} \cap S_{2}\right| \leq k / 2$ together imply $|X| \leq$ $2 k-\left|S_{1} \cap S_{2}\right|$. As the next theorem shows, these obviously necessary conditions are also sufficient (ONCAS).

Theorem 2. A set $X$ has a $k$-troika respecting $S_{1}, S_{2}$ (assume $\left|S_{1} \cup S_{2}\right|>k$ ) if and only if $|X| \leq\lfloor 3 k / 2\rfloor,\left|S_{1}\right| \leq k,\left|S_{2}\right| \leq k$ and $|X| \leq 2 k-\left|S_{1} \cap S_{2}\right|$

Proof: The necessity of these conditions have already been argued for. We prove that they are sufficient by considering two cases: $\left|S_{1} \cap S_{2}\right| \leq k / 2$ and $\left|S_{1} \cap S_{2}\right|>k / 2$. In the first case we can construct a 'balanced' $k$-tripartition $\left(T_{1}, T_{2}, T_{3}\right)$ where each partition class has at most $k / 2$ elements. For the vertices in $S_{1} \cap S_{2}$ we put them all in $T_{3}$. For the vertices in $S_{1} \backslash S_{2}$ we put up to $k / 2$ of them in $T_{1}$ and the remainder in $T_{3}$. For the vertices in $S_{2} \backslash S_{1}$ we put up to $k / 2$ of them in $T_{2}$ and the remainder in $T_{3}$. The conditions $|X| \leq\lfloor 3 k / 2\rfloor,\left|S_{1}\right| \leq k,\left|S_{2}\right| \leq k$, and $\left|S_{1} \cap S_{2}\right| \leq k / 2$ will ensure that each of $T_{1}, T_{2}, T_{3}$ constructed so far has at most $k / 2$ elements. The vertices in $X \backslash S_{1} \cup S_{2}$ are now put into $T_{1}, T_{2}$ or $T_{3}$ freely while simply ensuring that each partition class has at most $k / 2$ elements, which is doable since $|X| \leq\lfloor 3 k / 2\rfloor$ (note that if $k$ is odd then ' $\leq k / 2$ ', 'up to $k / 2$ ' and 'at most $k / 2$ ' is the same as $\leq\lfloor k / 2\rfloor$.)

We turn to the case $\left|S_{1} \cap S_{2}\right|>k / 2$. Let $f_{1}=k-\left(\left|S_{1} \cap S_{2}\right|+\left|S_{1} \backslash S_{2}\right|\right)$ and $f_{2}=$ $k-\left(\left|S_{1} \cap S_{2}\right|+\left|S_{2} \backslash S_{1}\right|\right)$. Note that $|X|-\left|S_{1} \cup S_{2}\right| \leq 2 k-\left|S_{1} \cap S_{2}\right|-\left|S_{1} \cup S_{2}\right|=f_{1}+f_{2}$ where the first inequality comes from $|X| \leq 2 k-\left|S_{1} \cap S_{2}\right|$. Thus we can partition $X \backslash S_{1} \cup S_{2}$ into $F_{1}$ and $F_{2}$ of sizes at most $f_{1}$ and at most $f_{2}$ respectively. The desired $k$-tripartition is then $T_{3}=S_{1} \cap S_{2}, T_{1}=\left(S_{1} \backslash S_{2}\right) \cup F_{1}, T_{2}=\left(S_{2} \backslash S_{1}\right) \cup F_{2}$.

Corollary 1. The smallest $k$ such that $X$ has a $k$-troika respecting $S_{1}, S_{2}$ is $\max \left\{\left|S_{1}\right|,\left|S_{2}\right|,\lceil 2|X| / 3\rceil, \min \left\{\left|S_{1} \cup S_{2}\right|,\left(\left\lceil|X|+\left|S_{1} \cap S_{2}\right|\right) / 2\right\rceil\right\}\right\}$ and can be computed in constant time given $\left|S_{1}\right|,\left|S_{2}\right|,|X|,\left|S_{1} \cap S_{2}\right|$.

Note that $\left|S_{1} \cup S_{2}\right|$ is easily found from $\left|S_{1}\right|,\left|S_{2}\right|,\left|S_{1} \cap S_{2}\right|$. The two terms inside the minimum covers the two cases where the resulting smallest $k$-troika $(A, B, C)$ has either $S_{1} \cup S_{2} \subseteq A$ or $S_{1} \subseteq A$ and $S_{2} \subseteq B$, respectively. Let us remark that for the interval graph algorithm the above Corollary suffices, since we then only deal with 2 minimal separators for each maximal clique.

\section{$4.2 \quad k$-Troikas respecting $q$ subsets}

We first consider the case of a set $X$ respecting three subsets $S_{1}, S_{2}, S_{3}$ and denote by $L$ the elements of $X$ not belonging to any subset and by $U_{i}, 1 \leq i \leq 3$ the elements belonging to $S_{i}$ only: $L=X \backslash\left(S_{1} \cup S_{2} \cup S_{3}\right), U_{1}=S_{1} \backslash\left(S_{2} \cup S_{3}\right)$, $U_{2}=S_{2} \backslash\left(S_{1} \cup S_{3}\right), U_{3}=S_{3} \backslash\left(S_{2} \cup S_{1}\right)$ (see Figure 4 in Appendix).

Lemma 3. $X$ has a $k$-troika $A, B, C$ with $S_{1} \subseteq A, S_{2} \subseteq B, S_{3} \subseteq C \Leftrightarrow$ the following system of linear equations in 5 non-negative integer variables $a, b, c, d$, e has a solution:

$$
\begin{aligned}
& a \leq\left|U_{1}\right| ; b \leq\left|U_{2}\right| ; c \leq\left|U_{3}\right| ; d+e \leq|L| \\
& \left|S_{3}\right|+\left|U_{2}\right|+a-b+d+e \leq k
\end{aligned}
$$




$$
\begin{aligned}
& \left|S_{1}\right|+\left|U_{3}\right|+|L|+b-c-e \leq k \\
& \left|S_{2}\right|+\left|U_{1}\right|+|L|-a+c-d \leq k
\end{aligned}
$$

Proof: $\Leftarrow$ : Partition $U_{1}$ into $L_{1}, F_{1}$ with $\left|L_{1}\right|=a$ and $\left|F_{1}\right|=\left|U_{1}\right|-a$. Partition $U_{2}$ into $F_{2}, R_{2}$ with $\left|F_{2}\right|=b$ and $\left|R_{2}\right|=\left|U_{2}\right|-b$. Partition $U_{3}$ into $R_{3}, L_{3}$ with $\left|R_{3}\right|=c$ and $\left|L_{3}\right|=\left|U_{3}\right|-c$. Partition $L$ into $F_{L}, R_{L}, L_{L}$ with $\left|F_{L}\right|=d$ and $\left|R_{L}\right|=e$ and $\left|L_{L}\right|=|L|-d-e$.

Then let $A=S_{1} \cup L_{3} \cup F_{2} \cup F_{L} \cup L_{L}$, let $B=S_{2} \cup R_{3} \cup F_{1} \cup F_{L} \cup R_{L}$, and let $C=S_{3} \cup L_{1} \cup R_{2} \cup L_{L} \cup R_{L}$.

The system of equations guarantee that the cardinalities of $A, B, C$ are at most $k$, and by construction we have $A \cup B=B \cup C=A \cup C=X$ and $S_{1} \subseteq A, S_{2} \subseteq$ $B, S_{3} \subseteq C$.

$\Rightarrow$ : Note that if $X$ has the desired $k$-troika then it has one with $A \cap B \cap C=$ $S_{1} \cap S_{2} \cap S_{3}$. Let $L_{1}=C \cap U_{1}$, let $F_{1}=B \cap U_{1}$, let $F_{2}=A \cap U_{2}$, let $R_{2}=C \cap U_{2}$, let $R_{3}=B \cap U_{3}$, and let $L_{3}=A \cap U_{3}$. Furthermore, let $F_{L}=L \cap A \cap B$, let $L_{L}=L \cap A \cap C$, and let $R_{L}=L \cap B \cap C$.

It follows that $A=S_{1} \cup L_{3} \cup F_{2} \cup F_{L} \cup L_{L}$, that $B=S_{2} \cup R_{3} \cup F_{1} \cup F_{L} \cup R_{L}$, and $C=S_{3} \cup L_{1} \cup R_{2} \cup L_{L} \cup R_{L}$.

Since the cardinalities of $A, B, C$ are at most $k$ we must have $a=\left|L_{1}\right|, b=$ $\left|F_{2}\right|, c=\left|R_{3}\right|, d=\left|F_{L}\right|, e=\left|R_{L}\right|$ a solution to the system of equations.

The only other possibility is that the union of two of the subsets is at most $k$ and in this case we may appeal to the conditions for respecting two subsets, giving:

Lemma 4. $X$ has a k-troika respecting $S_{1}, S_{2}, S_{3} \Leftrightarrow$ it has one satisfying the conditions of Lemma 3 or it has one where either $S_{1} \cup S_{2}, S_{3}$ or $S_{1} \cup S_{3}, S_{2}$ or $S_{2} \cup S_{3}, S_{1}$ satisfies the conditions of Lemma 2.

To respect $q>3$ subsets we simply note that since each subset must be contained in one of the three parts of the $k$-troika, there must exist a partition of the subsets into three classes such that every subset in the same class is contained in the same part.

Theorem 3. $X$ has a $k$-troika respecting $S_{1}, S_{2}, \ldots, S_{q} \Leftrightarrow$ there exists a partition of $\{1,2, \ldots, q\}$ into three classes $P_{1}, P_{2}, P_{3}$ such that by Lemma $4 X$ has a $k$-troika respecting the 3 subsets $W_{1}=\bigcup_{i \in P_{1}} S_{i}, W_{2}=\bigcup_{i \in P_{2}} S_{i}, W_{3}=\bigcup_{i \in P_{3}} S_{i}$.

Since a set of size $q$ has $3^{q}$ partitions into three classes we have:

Corollary 2. In time $O\left(\right.$ poly $\left.(|X|) 3^{q}\right)$ we can decide if a set $X$ has a $k$-troika respecting subsets $S_{1}, S_{2}, \ldots, S_{q}$.

\section{Algorithms computing branchwidth}

Throughout this section $G$ is a chordal graph with $m$ edges, $n$ vertices, maximal cliques $\left\{X_{1}, X_{2}, \ldots X_{q}\right\}$, having a clique-tree $T_{G}$ with nodes $\{1,2, \ldots, q\}$ such that node $i$ corresponds to maximal clique $X_{i}$. Mazoit [12] conjectured that branchwidth is computable in polynomial-time for any chordal graph given with a clique tree having polynomially many subtrees. We will prove his conjecture, but along the 
way we also give a fast algorithm for the case of interval graphs, i.e. when the clique tree is a path. We first define a merged supergraph of $G$ which is obtained by taking certain sets of maximal cliques that are connected in $T_{G}$ and merging each set into a larger clique.

Definition 6. $H$ is a merged supergraph of $G$ if there exists a partition of $T_{G}$ into subtrees $\left\{H_{1} \ldots H_{h}\right\}$ (each node $j \in V\left(T_{G}\right)$ belongs to one and only one subtree $\left.H_{i}\right)$ such that the set of maximal cliques in $H$ is: $\left\{X_{i}^{\prime}=\cup_{j \in T_{i}} X_{j}\right\}(1 \leqslant i \leqslant h)$.

It is straightforward to see that a merged supergraph $H$ of a chordal graph $G$ is chordal with clique-tree $T_{H}$ built by making maximal cliques $X_{i}^{\prime}$ and $X_{j}^{\prime}$ adjacent iff $H_{i}$ and $H_{j}$ contains two adjacent nodes of $T_{G}$, in other words $T_{H}$ is a minor of $T_{G}$. We first show that to find the branchwidth $k$ of $G$ it suffices to search for $k$-good chordal graphs among the merged supergraphs of $G$.

Lemma 5. Let $G$ be a chordal graph of bw $(G)=k$ and let $H$ be a $k$-good chordal supergraph of $G$. Let $X$ be a maximal clique of $G$ whose neighboring maximal cliques in $T_{G}$ are $X_{1}, X_{2} \ldots X_{l}$. If $X$ does not have a $k$-troika respecting the minimal separators in $X$, then there exists $X_{i}(1 \leqslant i \leqslant l)$ such that $X_{i} \cup X$ is a clique in $H$.

Proof: Let $S_{i}=X \cap X_{i}(1 \leqslant i \leqslant l)$. Let us first recall that as $X$ and $X_{i}$ are adjacent maximal cliques $S_{i}$ is a minimal separator and moreover any minimal separator contained in $X$ is a subset of some such $S_{i}(1 \leqslant i \leqslant l)$. Therefore it suffices that the $k$-troika respects $S_{1}, S_{2}, \ldots, S_{l}$.

Proof by contradiction. $S_{i}$ is a minimal $a, b$-separator, for any $a \in X_{i} \backslash X$ and $b \in X \backslash X_{i} . X$ is a subset of some maximal clique $X^{\prime}$ of $H$. Assume $X_{i} \cup X$ is not a clique of $G^{\prime}$. As $H$ is chordal, there must exist a subset $S_{i}^{\prime}$ of $X$ which is a miminal $a, b$-separator $S_{i}^{\prime}$ for some $a \in X_{i} \backslash X^{\prime}$ and $b \in X^{\prime} \backslash X_{i}$. Moreover, $S_{i}$ has to be included in $S_{i}^{\prime}$, since otherwise we would have an $a, b$-path avoiding $S_{i}^{\prime}$. Likewise, for any $1<i \leqslant l$, unless $X \cup X_{i}$ is a clique we find $S_{i}^{\prime} \subset X^{\prime}$ a minimal separator containing $S_{i}$. As $X \subseteq X^{\prime}, S_{i} \subseteq S_{i}^{\prime}$ for $1 \leqslant i \leqslant l, X^{\prime}$ cannot have a $k$-troika respecting $S_{1}^{\prime} \ldots S_{l}^{\prime}$ as long as $X$ did not have one respecting $S_{1} \ldots S_{l}$, a fact that follows from Theorem 3 . This contradicts the fact that $H$ is a $k$-good chordal graph.

Lemma 6. A chordal graph $G$ has bw $(G) \leqslant k \Leftrightarrow$ there exists a $k$-good chordal graph $H$ that is a merged supergraph of $G$.

Proof: $\Leftarrow$ : By Theorem 1 the existence of a $k$-good chordal graph $H$ that is a merged supergraph of $G$ implies that $b w(G) \leqslant k$.

$\Rightarrow$ : By induction on the number $q$ of maximal cliques of $G$. If $G$ has at most 2 maximal cliques, then Lemma 5 establishes the claim. Assume by induction that the property holds for any chordal graph of branchwidth $k$ having $q \geq 2$ maximal cliques. If $G$ is not a $k$-good chordal graph, then it has a maximal clique $X$ which does not have a $k$-troika respecting the minimal separators $X_{1} \cap X, X_{2} \cap$ $X, \ldots, X_{l} \cap X$, where $X_{1} \ldots X_{l}$ are the neighbors of $X$ in the clique tree $T_{G}$. Since $G$ has branchwidth $k$ it has some $k$-good chordal supergraph in which, by Lemma 5 , some neighbor $X_{j}(1 \leq j \leq l)$ has been merged with $X$ into a bigger clique. But then consider the merged supergraph of $G$ arising from merging exactly $X$ and $X_{j}$ 
into one clique. It has $q-1$ maximal cliques and by the induction hypothesis there is a $k$-good chordal graph $H$ which is a merged supergraph of $G^{\prime}$ and therefore also of $G$.

\subsection{Branchwidth of interval graphs}

A graph is an interval graph iff it enjoys a consecutive clique arrangement (cca) that is an ordering of its maximal cliques $\mathcal{C}=\left(X_{1}, \ldots X_{q}\right)$ such that for any vertex $x$, the maximal cliques containing $x$ occur consecutively. From any linear time interval graph recognition algorithm such a cca can be computed (see e.g. [3]). It is well known that for any $1<i \leqslant q$, the set $S_{i}=X_{i-1} \cap X_{i}$ is a minimal separator. Let $S_{1}=S_{q+1}=\emptyset$ be dummy separators. Let us denote by $X_{i, j}=\cup_{i \leqslant g \leqslant j} X_{g}$ $(1 \leqslant i \leqslant j \leqslant q)$ a merged set of consecutive cliques.

Given a cca $\mathcal{C}_{G}=\left(X_{1} \ldots X_{q}\right)$ of an interval graph $G$, a merged supergaph $H$ of $G$ has caa $\mathcal{C}_{H}=\left(X_{1}^{\prime} \ldots X_{h}^{\prime}\right)$ with $h \leq q$ such that for any $1 \leqslant i \leqslant h, X_{i}^{\prime}=X_{l_{i}, r_{i}}$ with $l_{1}=1, l_{i}=r_{i-1}+1$ for $i>1$ and $r_{h}=q$. Note that a merged supergraph of an interval graph is also an interval graph.

Our algorithm first computes for each pair $1 \leq i \leq j \leq q$ the smallest value $K[i, j]$ such that if we merge the consecutive cliques $X_{i, j}$ into one big clique, it will have a $K[i, j]$-troika respecting $S_{i}$ and $S_{j+1}$. Then by simple dynamic programming it computes the best way of merging various such sets into a merged supergraph, see Figure 1. Incrementally, in step $j$, we optimize over the possible cutoff points $1 \leq$ $i \leq j$ that define the 'rightmost' merged set of cliques $X_{i, j}$. We prove correctness before considering the running time.

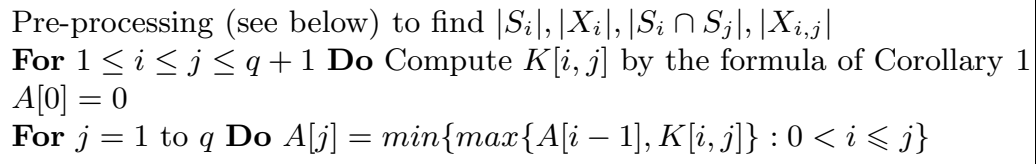

Fig. 1. Computation of $b w(G)=A[q]$ for interval graph $G$.

Theorem 4. The computed value $A[q]$ is the branchwidth of interval graph $G$.

Proof: Let us prove by induction that, for $1 \leqslant i \leqslant q, A[i]=b w\left(G_{i}\right)$ where $G_{i}$ is the graph induced by $X_{1, i}$ with an extra dummy vertex $x_{i}$ adjacent to $S_{i+1}$. By Corollary $1 K[i, j]$ is the minimum such that set $X_{i, j}$ has a $K[i, j]$-troika respecting $S_{i}$ and $S_{j+1}$. As $A[1]=K[1,1], X_{1}$ has a $A[1]$-troika respecting $S_{2}$. Therefore $\left\{x_{1}\right\} \cup S_{2}$ also has a $A[1]$-troika respecting $S_{2}$. Theorem 1 implies that $b w\left(G_{1}\right)=A[1]$. Assume that $A[j-1]=b w\left(G_{j-1}\right)$ for $j>1$. Let $H_{j}$ be the merged supergraph of $G_{j}$ such that $b w\left(G_{j}\right)=b w\left(H_{j}\right)$. Then by Lemma 5 the maximal clique $X_{j}$ is contained in $H_{j}$ in a maximal clique $X^{\prime}=X_{i, j}$ for some $1 \leqslant i \leqslant j$. It therefore follows from Lemma 6 , that $b w\left(G_{j}\right) \leqslant \max \{A[i-1], K[i, j]\}$ for any $1 \leqslant i \leqslant j$ and thus $b w\left(G_{j}\right)=A[j]$. We proved that $b w\left(G_{q}\right)=A[q]$. Since $G_{q}$ is the union of two connected components, the first one being $G$ itself and the second an isolated vertex $x_{q}, b w(G)=b w\left(G_{q}\right)$. 
By Corollary 1 the computation of matrices $K$ and $A$ takes time $O\left(q^{2}\right)$ if the values $\left|S_{i}\right|,\left|X_{i}\right|,\left|S_{i} \cap S_{j+1}\right|$, and $\left|X_{i, j}\right|$ can be accessed in $O(1)$ time. We now show that these values can be made available in array locations $S[i], X[j], S[i, j], X[i, j]$ by pre-processing stage. Any interval graph recognition algorithm [3] is able to ouput in $O(n+m)$ time the size $X[i]=\left|X_{i}\right|$ of any maximal clique and $S[i]=\left|S_{i}\right|$ of any minimal separator, and also for any vertex $x$ the range $[\operatorname{Left}(x), \operatorname{Right}(x)]$ of consecutive cliques containing $x$. From those values, assuming for any $1 \leqslant i \leqslant q$ $X[i, i]=\left|X_{i}\right|$, we have for $i+1 \leqslant j \leqslant q, X[i, j]=X[i, j-1]+X[j]-S[j]$. To find the values $S[i, j]=\left|S_{i} \cap S_{j+1}\right|$ fast, we first compute the intermediary $q \times q$-matrix $M$ such that for $i<j, M[i, j]=\left|\left(S_{i} \cap S_{j}\right) \backslash S_{j+1}\right|$. Since $\left|S_{i} \cap S_{j}\right|=$ $\sum_{h \leqslant j}\left|\left(S_{i} \cap S_{j}\right) \backslash S_{j+1}\right|$, the array $S[i, j]$ can be computed as follows:

$$
\begin{aligned}
& \text { Initialize each entry of } M[i, j] \text { to } 0 \\
& \text { For any } S_{i}(2 \leqslant i \leqslant q) \text { and } x \in S_{i} \text { Do If } \operatorname{Right}(x)=j \text { Then add } 1 \text { to } M[i, j] \\
& \text { For } i=2 \text { to } q \text { Do } \mid \begin{array}{l}
S[i, q]=M[i, q] \\
\text { For } j=q-1 \text { downto } i \text { Do } S[i, j]=S[i, j+1]+M[i, j]
\end{array}
\end{aligned}
$$

As the sum of the sizes of the minimal separators of an interval graph is bounded by $m$, this preprocessing requires $O\left(m+n+q^{2}\right)$ time. We have shown:

Theorem 5. Branchwidth of an interval graph $G=(V, E)$ on $m$ edges, $n$ vertices and $q \leq n$ maximal cliques can be computed in time $O\left(n+m+q^{2}\right)$.

\subsection{Clique trees with polynomial number of subtrees}

For a subtree $T^{\prime}$ of a tree $T$ we define its connection points as the pairs of vertices $a_{1} b_{1}, a_{2} b_{2}, \ldots, a_{p} b_{p}$ such that $a_{i} b_{i}$ is an edge of $T$ with $a_{i} \in T^{\prime}$ and $b_{i} \in T \backslash T^{\prime}$. Assume clique tree $T_{G}$ of chordal graph $G$ has a polynomial number of subtrees $T_{1}, T_{2}, \ldots, T_{t}$, ordered by size. Let $T_{i}$ have connection points $a_{1} b_{1}, a_{2} b_{2}, \ldots, a_{p} b_{p}$. Define the connection separators of $T_{i}$ to be $S_{j}=X_{a_{j}} \cap X_{b_{j}}$ for $1 \leq j \leq p$, where $X_{a_{j}}, X_{b_{j}}$ are the maximal cliques of $G$ corresponding to tree nodes $a_{j}, b_{j}$. Define $K[i]$ to be True if $V\left(T_{i}\right)$ has a $k$-troika respecting the connection separators $S_{1}, S_{2}, \ldots, S_{p}$ of $T_{i}$. The following algorithm will in polynomial time decide if $G$ has branchwidth at most $k$ :

For $i=1$ to $t$ Do Compute boolean $K[i]$ by the system of equations of Theorem 3 $A[i]=T$ if $K[i]=T$ or if $\exists e \in E\left(T_{i}\right)$ with $A\left[e_{1}\right]=T$ and $A\left[e_{2}\right]=T$ for subtrees $T_{e_{1}}, T_{e_{2}}$ of $T_{i} \backslash e$; otherwise $A[i]=F$

Fig. 2. Branchwidth of $G \leq k$ iff $A[t]=T$

Theorem 6. For a chordal graph $G$ given with a clique tree having a polynomial number $t$ of subtrees the above algorithm will in polynomial time decide if branchwidth of $G$ is at most $k$. 
Proof: We first argue for the timing: Since clique tree $T_{G}$ on $q$ nodes has a number of subtrees that is polynomial in $q$ then the number of connection points $p$ for any subtree $T_{i}$ must be logarithmic in $q \leq n$ (a subtree with $p$ leaves has itself at least $2^{p}$ subtrees.) Corollary 2 tells us that we can then in time polynomial in $n$ decide if $V\left(T_{i}\right)$ has a $k$-troika respecting its $p$ subsets.

Now the correctness: Let $G_{i}$ be the graph induced by $\bigcup_{j \in V\left(T_{i}\right)} X_{j}$ with $p$ extra dummy vertices adjacent to each of the $p$ connection separators $S_{1}, S_{2}, \ldots, S_{p}$ of $T_{i}$. We prove by induction on the size of the subtrees that, for $1 \leqslant i \leqslant t, A[i]=$ True iff $b w\left(G_{i}\right) \leq k$. By Theorem $3 K[i]$ is True iff the set $V\left(T_{i}\right)$ has a $K[i]$-troika respecting its connection separators. Thus, $A[i]$ is certainly correct if $T_{i}$ has no edge. Assume $A[i]$ correct for all subtrees on $f$ edges. For some $T_{i}$ on $f+1$ edges, if $G_{i}$ has branchwidth at most $k$ then some merged supergraph of $G_{i}$ is a $k$-good chordal graph, by Lemma 6 . Either this merged supergraph has $V\left(G_{i}\right)$ as one big clique, in which case $K[i]$ is True, or there is an edge $e$ of $T_{i}$ such that for the two subtrees $T_{e_{1}}$ and $T_{e_{2}}$ of $T_{i} \backslash e$ we have $b w\left(G_{e_{1}}\right) \leq k$ and $b w\left(G_{e_{2}}\right) \leq k$. Since $T_{e_{1}}$ and $T_{e_{2}}$ have at most $f$ edges each, this is correctly recorded by $A\left[e_{1}\right]$ and $A\left[e_{2}\right]$.

\section{References}

1. H.L. Bodlaender and D.M. Thilikos. Constructive linear time algorithms for branchwidth. 627-637, ICALP'97

2. H.L. Bodlaender and D.M. Thilikos. Graphs with branchwidth at most three. Journal of Algorithms, 32:167-194, 1999.

3. K. Booth and G. Lueker. Testing of the consecutive ones property, interval graphs, and graph planarity testing using PQ-tree algorithms. Journal of Computer and System Sciences, 13:335-379, 1976.

4. W. Cook and P.D. Seymour. Tour merging via branch-decompositions. Journal on Computing, 15:233-248, 2003.

5. E. Demaine, F. Fomin, M. Hajiaghayi, and D.M. Thilikos. Fixed-parameter algorithms for (k,r)-center in planar graphs and map graphs. 829-844, ICALP'03

6. F. Fomin and D. Thilikos. Dominating sets in planar graphs: Branch-width and exponential speedup. 168-177, SODA'03

7. F. Fomin and D. Thilikos. A simple and fast approach for solving problems on planar graphs. STACS'04

8. F. Fomin and D. Thilikos. Fast parameterized algorithms for graphs on surfaces: Linear kernel and exponential speedup ICALP'04

9. F. Gavril. The intersection graphs of subtrees in trees are exactly the chordal graphs. Journal of Combinatorial Theory Series B, 16:47-56, 1974.

10. M.C. Golumbic. Algorithmic Graph Theory and Perfect Graphs. Acad. Press, 1980.

11. T. Kloks, J. Kratochvil, and H. Müller. New branchwidth territories. 173-183, STACS'99. To appear Discrete Applied Mathematics.

12. F. Mazoit. A general scheme for deciding the branchwidth. Technical Report RR200434, LIP - École Normale Supérieure de Lyon, 2004, presented at 6th Journées Graphes et Algorithmes, Grenoble, September 2004 http://www.ens-lyon.fr/LIP/Pub/Rapports/RR/RR2004/RR2004-34.pdf.

13. N. Robertson and P.D. Seymour. Graph minors X: Obstructions to treedecomposition. Journal on Combinatorial Theory Series B, 52:153-190, 1991.

14. P.D. Seymour and R. Thomas. Call routing and the ratcatcher. Combinatorica, 14(2):217-241, 1994. 


\section{Appendix}
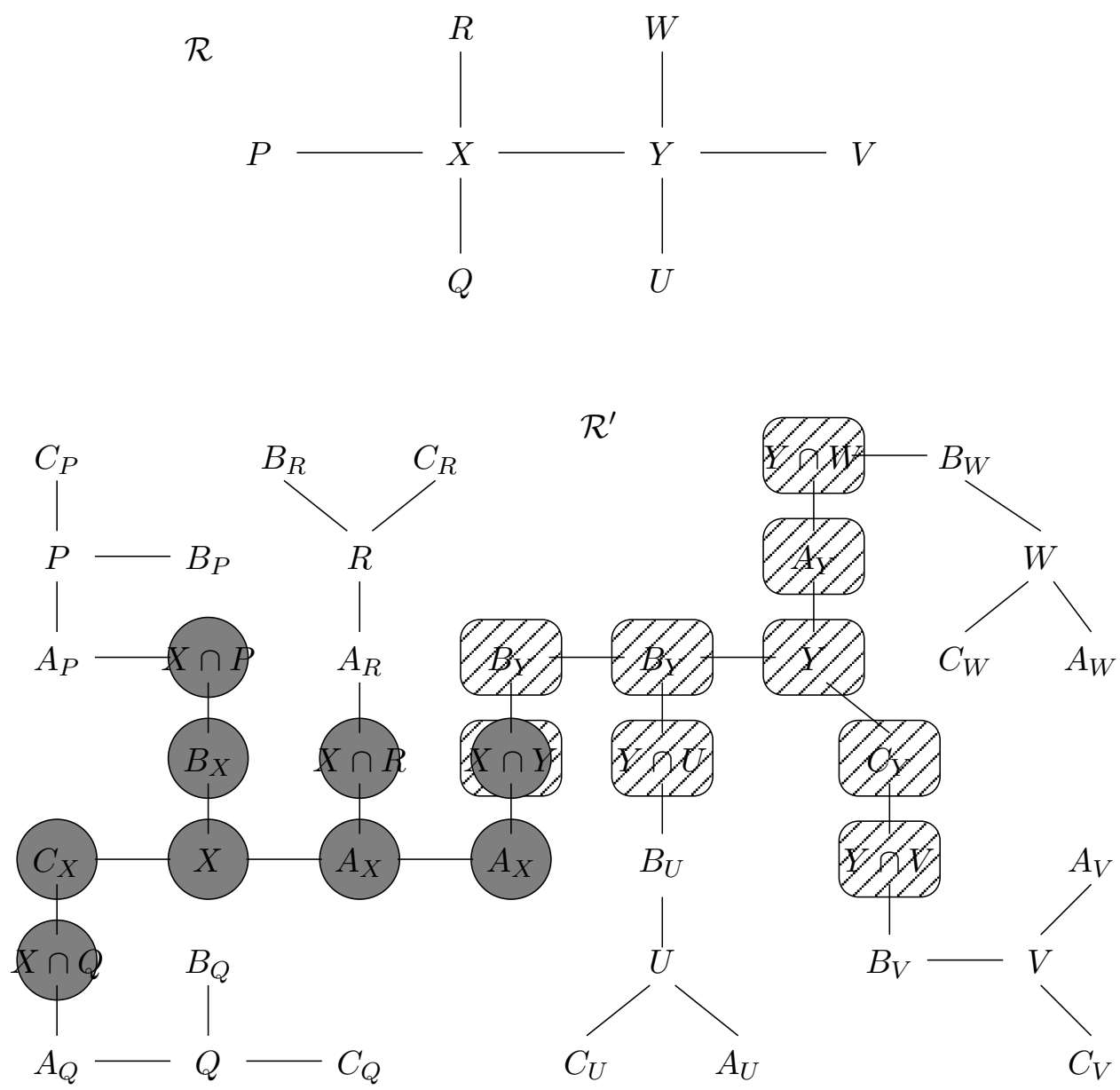

Fig. 3. Theorem 1. On top a clique tree of a $k$-good chordal graph $H$ with $k$-troika of any maximal clique $M$ being $\left(A_{M}, B_{M}, C_{M}\right)$. Below the constructed subtree-representation $R^{\prime}$ of edge-weight $k$ such that $H \subseteq E I\left(R^{\prime}\right)$. The square nodes correspond to the ternary subtree associated with clique $Y$ and the grey nodes to the ternary subtree associated to clique $X$. Both ternary subtrees share the leaf $X \cap Y$ where they connect. 


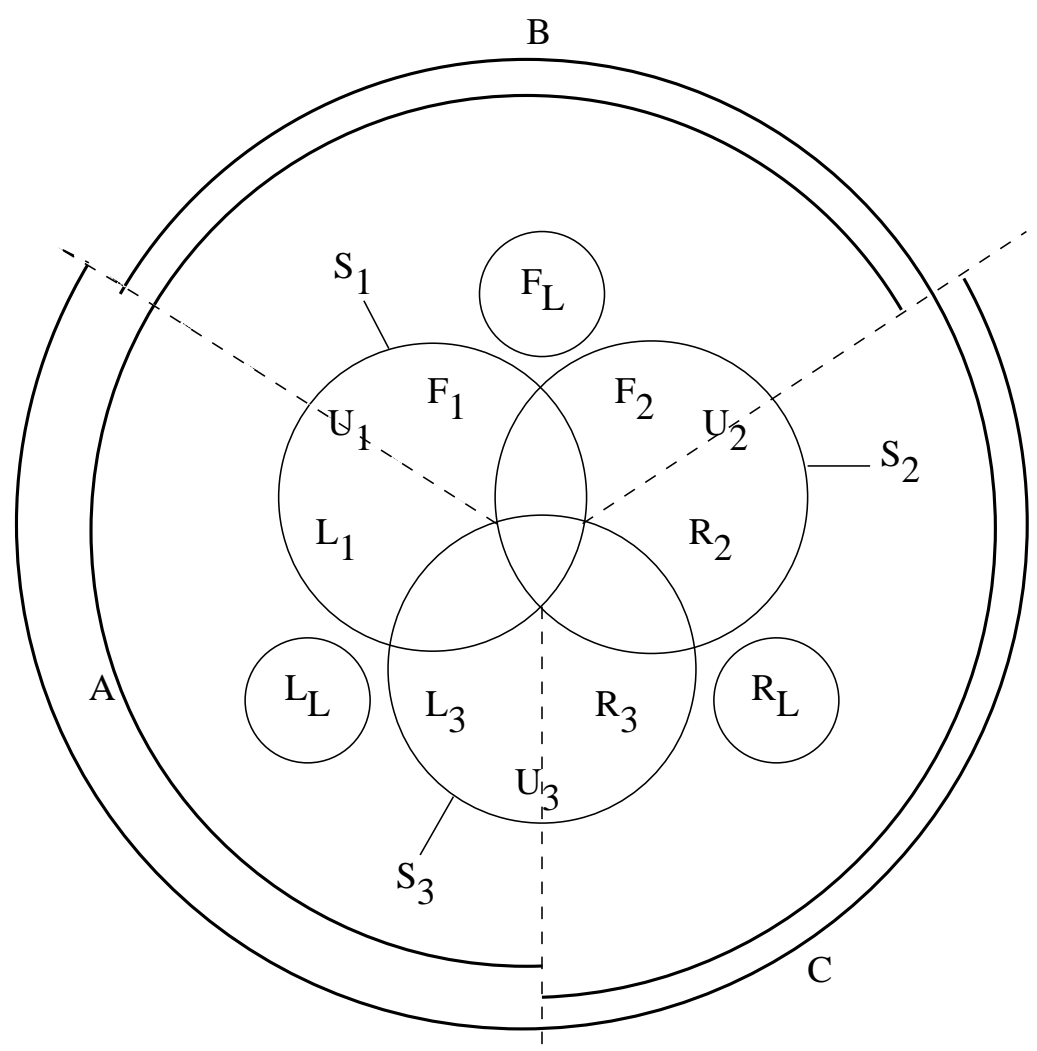

Fig. 4. Venn diagram of a set $X$ consisting of the 6 circles $S_{1}, S_{2}, S_{3}, F_{L}, L_{L}, R_{L}$. If $X$ has $k$-troika $(A, B, C)$ respecting $S_{1}, S_{2}, S_{3}$ then we may as well require $S_{1} \cap S_{2} \cap S_{3}=$ $A \cap B \cap C$. The sets $A, B, C$ are otherwise illustrated using the dotted lines at 2, 6 and 10 o'clock, e.g. $A$ contains elements between 6 and 2 o'clock. Elements belonging to only one of the $S_{i}$ sets are named $U_{i}$ and further partitioned in two parts by the dotted lines, as defined in subsection 4.2 . 